\title{
Seismic waves in the ionosphere
}

\author{
Philippe Lognonné ${ }^{1}$, Raphael Garcia ${ }^{1}$, François Crespon ${ }^{1}$, Giovanni Occhipinti ${ }^{1}$, Alam Kherani ${ }^{1}$ and Juliette Artru-Lambin ${ }^{2}$, \\ ${ }^{1}$ Institut de Physique du Globe de Paris, Saint Maur des Fossés • France \\ ${ }^{2}$ Centre National d'Etudes Spatiales, Toulouse • France
}

40 years after the beginning of Earth observation from space, the monitoring of Earth's seismic activity and the recording of the seismic waves generated by quakes is exclusively done by global and regional seismic networks. Contrary to geomagnetism or gravimetry, seismology does not yet benefit from data acquired from space, and has to deal with a non-homogeneous coverage of seismic stations. In particular, the oceans, which cover more than $70 \%$ of the Earth surface, are almost devoid of measurements.

Seismic data are indeed based on the detection of very low ground displacements: even at the most noisy frequency, $0.15 \mathrm{~Hz}$, associated with a global seismic noise generated by the oceanic waves, the amplitude of the ground displacement noise is in the range of 0.1-10 micrometers and good signal to noise seismic waves have amplitudes of a hundred to a thousand times higher... Such amplitudes are far below the resolution of any space altimetry methods, especially due to the Earth's atmosphere and its interaction with the radio waves or with visible light. Remote detection of the seismic signals from space seems hopeless...

However, the atmosphere is also affected by waves, and turns out to offer a possible solution to the problem. After an earthquake, seismic waves generate vertical and horizontal motion of the surface of the Earth. In some case, tsunami or oceanic gravity waves are also generated and produce surface oscillations. By continuity of vertical displacement at the surface, the atmosphere is then forced to move with the same vertical velocity as the ground surface. The perturbation propagates upward as an atmospheric wave and produces pressure and temperature variations, and oscillations of the atmospheric layers. Can these atmospheric waves be detected remotely? Let us look at the amplitudes...
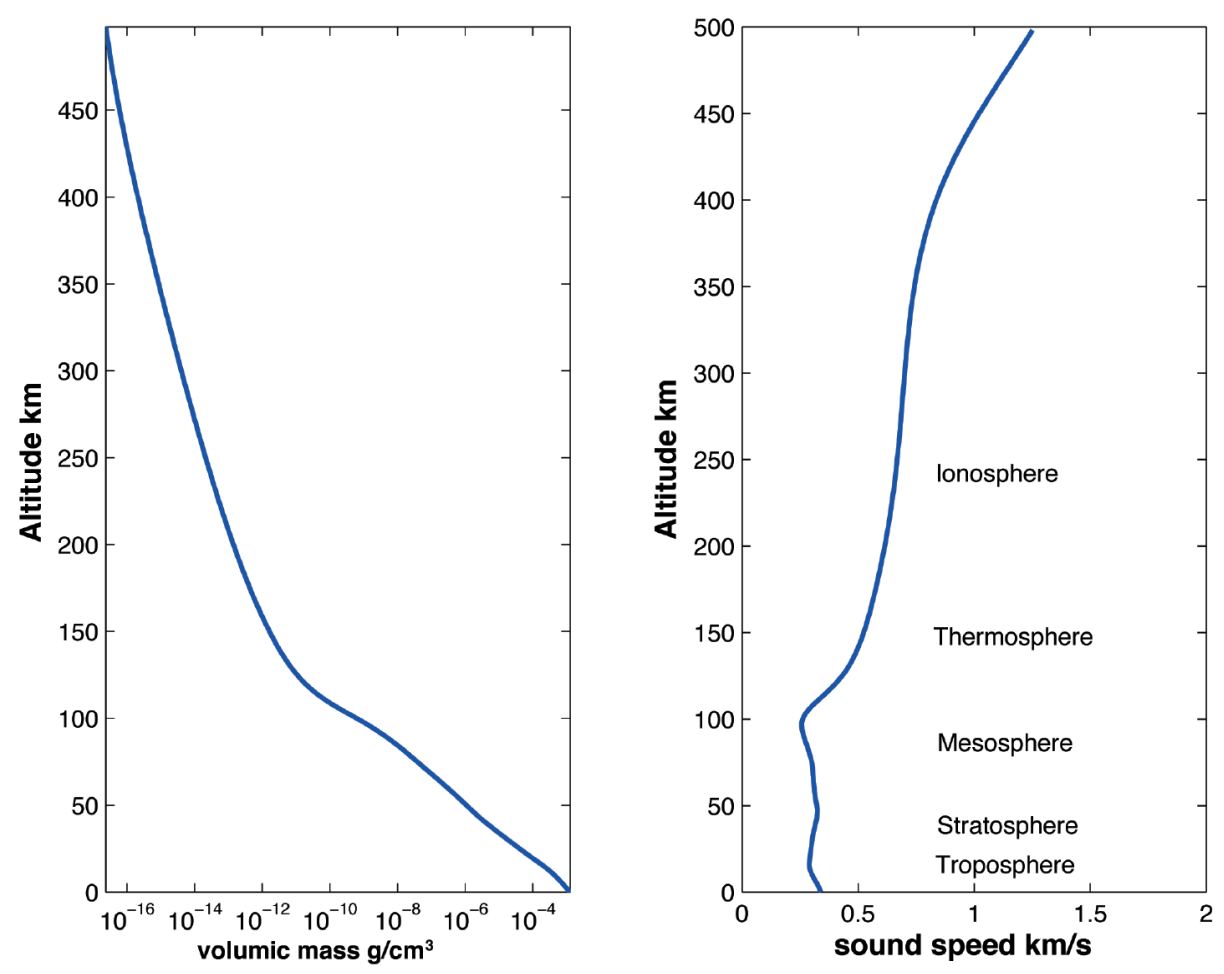

The seismic waves with the largest ground amplitude are the surface waves and especially the Rayleigh waves. They propagate along the Earth's surface, in the crust or upper mantle, with velocities ranging from 3 to $4 \mathrm{~km} / \mathrm{s}$. For large and superficial quakes, their displacement amplitude, even at an epicentral distance of 10000 $\mathrm{km}$, can reach several $\mathrm{mm}$ or even $\mathrm{cm}$, as was observed after the large Sumatra earthquake of December, 26, 2004. The propagation speed of the wave front being much larger than the sound speed in the atmosphere, the generated air waves propagate almost vertically from the surface location of the Rayleigh wave front. With their long periods ( $\mathrm{T}>10-20 \mathrm{~s}$ ), the infrasonic atmospheric waves are not attenuated by the atmospheric viscosity: they propagate with a constant kinetic energy and therefore, their amplitude grows exponentially as the inverse of the square root of density. As shown by Fig. 1, the density decays by 10 orders of magnitude between the ground and $200 \mathrm{~km}$ of altitude and amplification of $10^{5} \mathrm{can}$ therefore be encountered by air waves during their upward propagation. But the neutral atmosphere is not the only one to oscillate. A transfer is made by collision processes to the ionospheric ions and associated electrons, which oscillate almost in phase. Finally for quakes of magnitude 7 or more, the ionosphere electrons are oscillating with velocities of a few tens of $\mathrm{m} / \mathrm{s}$ and are displaced by a few hundred of meters. These forced ionospheric waves, with horizontal speed imposed by the true solid earth surface waves, are the target for remote sensing observation of the surface waves.

The first observation of ionospheric surface waves were obtained after a very large Alaskan quake in 1964. At that time, the ionosphere was monitored for the purpose of nuclear explosion detection, and both the theories and the instruments necessary for the interpretation of the atmospheric gravity waves generated by megatonic atmospheric explosions [1] had been developed, especially at the Seismological Laboratory of the California Institute of Technology. The pressure fluctuations generated by quakes are much smaller than those generated by typical gravity waves. The seismic source is indeed located in the solid earth and the coupling between the solid part and the atmosphere transfers only $10^{-4}$ to $10^{-5}$ of the energy [2]. Resonances are however found first near 3.7 $\mathrm{mHz}$, where spheroidal modes have up to $0.05 \%$ of their energy in the atmosphere and also near $4.4 \mathrm{mHz}$. These two maximums result from a wavelength matching of the Rayleigh

Fig. 1: Density and sound speed profile with altitude in the Earth atmosphere. The ionosphere is developing above $120 \mathrm{~km}$ of altitude and a maximum of ionization is reached at $350-400 \mathrm{~km}$ of altitude. 
waves with the mesospheric wave guide [2] and can lead to typical bi-chromatic seismic signals after volcanic atmospheric explosions [3].

Even for a magnitude 8 quake, the pressure fluctuations reach only a few microbars at the surface and must be recorded with complex measurements systems. The Earth atmosphere is however acting as a natural amplifier and leads to large signals at $150 \mathrm{~km}$ or more of altitude in the ionosphere, for quakes of magnitude 7 or larger. The ionospheric oscillations can be remotely sounded by a Doppler sounder [4]. The principle of the Doppler sounder is to send an electromagnetic wave in the range of 1 to $15 \mathrm{MHz}$. The emitted wave reaching the plasma modifies its propagation as the electron density increases and will be fully reflected at a given altitude, where the plasma frequency (a function of local electron density) matches the radio wave frequency. If the reflecting layer is oscillating vertically, a Doppler effect is indeed observable in the
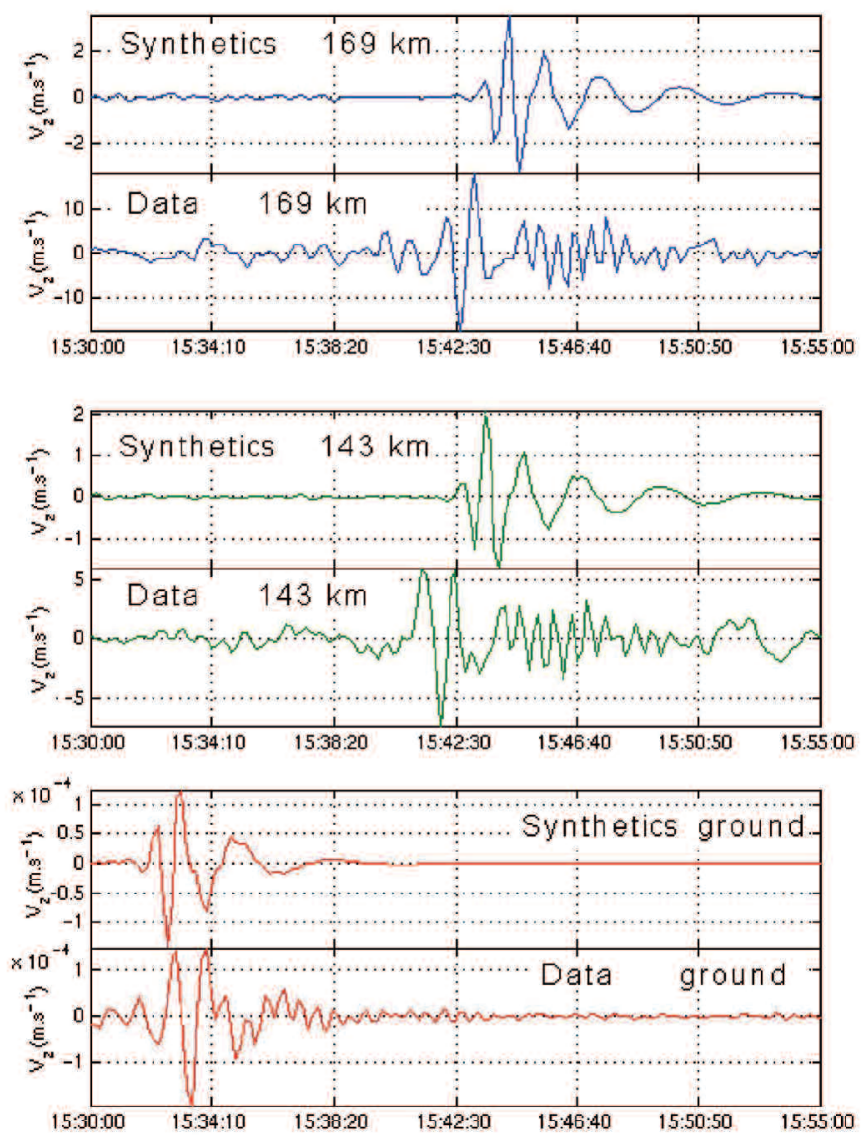

$\Delta$ Fig. 2: These signals correspond to the ionospheric vertical velocity. They are recorded by the Doppler sounder of CEA, at 143 and $169 \mathrm{~km}$ of altitude, by using the reflection of two radio waves at 3.849 and $4.624 \mathrm{MHz}$ respectively. Data were recorded after a $\mathrm{Mw}=8$ quake in the South Indian Ocean, on June 18, 2000. The bottom trace corresponds to the data recorded by a seismometer. For all data, synthetics are computed by using a spherically symmetric Earth model, for both the solid Earth or its atmosphere. Most of the differences at the ground are associated with the 3D structure of the Earth, which is not taken into account in the synthetics. Differences in the amplitude of the ionospheric waves are probably due to a non-correct viscosity profile in the atmosphere, such data being badly known at high altitude. Data from CEA-DASE are reprinted from [4]. reflected signal. The ground-based Doppler sounding can therefore follow the oscillations ionospheric layers up to $350 \mathrm{~km}$ of altitude, where the maximum of ionization is reached. Modern instruments can detect vertical velocities of a few 10 of $\mathrm{cm} / \mathrm{s}$, enabling therefore the detection of all quakes with magnitude greater than 7 (Fig. 2).

If these techniques are paving the way of a seismic remote sensing of the signals, they however remain single point measurements and do not allow the wavefield to be imaged. A second step was therefore necessary toward such 2D or 3D mapping of the seismic waves in the atmosphere. Our team, with the French SME Noveltis, has used for this goal the dense GPS networks, located either in California, Japan or Europe. With about 1200 receivers the Japan network is the densest and as each receiver can see from 6 to 10 satellites, this network provides about 10000 ionospheric sounding each second. These data allow, by tomographic techniques, the airwaves in $2 \mathrm{D}$ and $3 \mathrm{D}$ to be captured, through their perturbations of the electronic content of the ionosphere [5]. Typically, these perturbations reach a few percent of the electron density for quakes with magnitude larger than 8 (Fig. 3). The vertical and horizontal propagation of the wave front can then be studied. By using the Southern California GPS network, the surface waves of the November, 3, $2002(\mathrm{M}=8.2)$ quake were clearly detected between an altitude of 200 and $400 \mathrm{~km}$ (see the movies of the ionospheric perturbations and more information on the Spectre project [6]). Estimates of the horizontal group velocity has been obtained and successfully compared with the velocity from 3D models of the Earth lateral variations [7]: we can even imagine using these techniques for measuring the seismic velocities of the Earth upper mantle and crust, especially in locations where seismometers cannot be easily deployed (Fig. 4). Although on Earth this technique would never provide the same quality of seismic data as a seismic network, it can be the unique way to obtain seismic data on planets too hostile for the deployment of long lived seismic stations. Venus is the best example [8]. In addition, the coupling strength is proportional to the acoustic impedance of the atmosphere, equal to $\rho c$ where $\rho$ is the density and $c$ the acoustic speed. As the atmospheric density at the surface of Venus is about $60 \mathrm{~kg} / \mathrm{m}^{3}$ and the acoustic velocity is slightly higher $(410 \mathrm{~m} / \mathrm{s})$ than on Earth, this leads to an acoustic impedance about 60 times greater than on Earth, where the atmospheric density is $1.2 \mathrm{~kg} / \mathrm{m}^{3}$. Moreover, at $50 \mathrm{~km}$ of altitude, where the Venus pressure is comparable to Earth ground pressure, the decrease by almost 2 orders of magnitude of the density leads already to an amplification of 10 of the waves. Consequently, Venus quakes will generate atmospheric infrasonic waves with amplitudes 600 larger than on the Earth surface. This profitable effect gives a unique opportunity for a future Venus quakes detection by a satellite sounding the Venus ionosphere.

\section{Let's however go back to Earth...}

Tsunamis can also be detected with such a remote sensing approach. Tsunamis are surface gravity waves that propagate for great distances in the oceans, usually triggered by earthquakes or landslides. In the open ocean, their long wavelengths (typically $200 \mathrm{~km}$ ), long periods (20 minutes) and small amplitudes (a few to $50 \mathrm{~cm}$ for the gigantic event of December, 26) make their detections very challenging with the GPS buoy systems or ocean bottom pressure sensors. Recently, satellite altimetry has proved to be capable of measuring the sea surface variation in the case of large tsunamis [9] including for the recent Sumatra, December 26 tsunami [10], but these techniques do not allow a real time imaging of the oceanic wave. 
As for surface waves, early theoretical works in the 1970s predicted that atmospheric gravity waves are generated in the wake of a tsunami [11]. If one hour is, for the gravity wave, the time to reach the ionosphere (versus $\sim 10$ minutes for seismic-acoustic waves), after this delay the ionospheric perturbation follows the tsunami front and, as for the seismic waves, the atmospheric oscillations are amplified with altitude. It should be noted moreover that, due to their much shorter wavelength and period, the surface noise of ocean swell does not produce significant upward propagating waves in the atmosphere: the atmosphere acts as a filter, enhancing the long wavelength tsunami perturbation over other sources.

The first observation had however to wait almost 30 years. It was performed after the Peru, June 32, 2001 tsunami [12]. The tsunami arrival was observed on Japanese tide gauges between 20 and 22

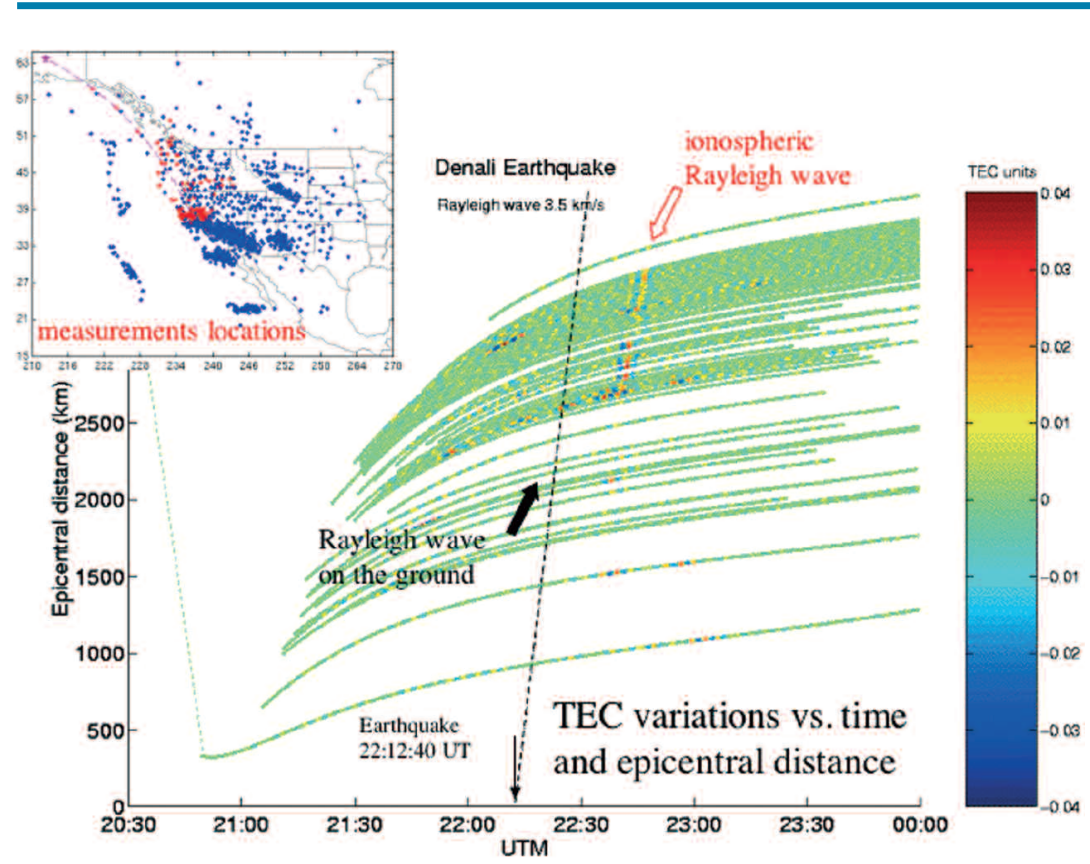

4 Fig. 3: TEC time series from satellite 26, plotted as a function of time and distance to the epicenter. The TEC data were obtained from the difference of the two GPS carriers propagation times and then band-pass filtered between $150 \mathrm{~s}$ and $350 \mathrm{~s}$, with a central period of $225 \mathrm{~s}$. Each trace corresponds to the TEC obtained with a given GPS station at the sub-ionospheric point. The satellite elevation is about 60-65 degrees at the time of signal observation. The positions of the sub-ionospheric points are obtained from reprocessed satellite coordinates with a $30 \mathrm{~s}$ resampling. The black dashed line represents a typical Rayleigh wave on the ground, with a mean velocity of $3.5 \mathrm{~km} / \mathrm{s}$. Differences in arrival time might be related to lateral variation. On the top left: Piercing points for ionospheric measurements from all receivers in California $(S C I G N+B A R D+I G S)$ and all satellites. The red star shows the epicenter location for the Denali earthquake and the red points are the position of the sub-ionospheric points.
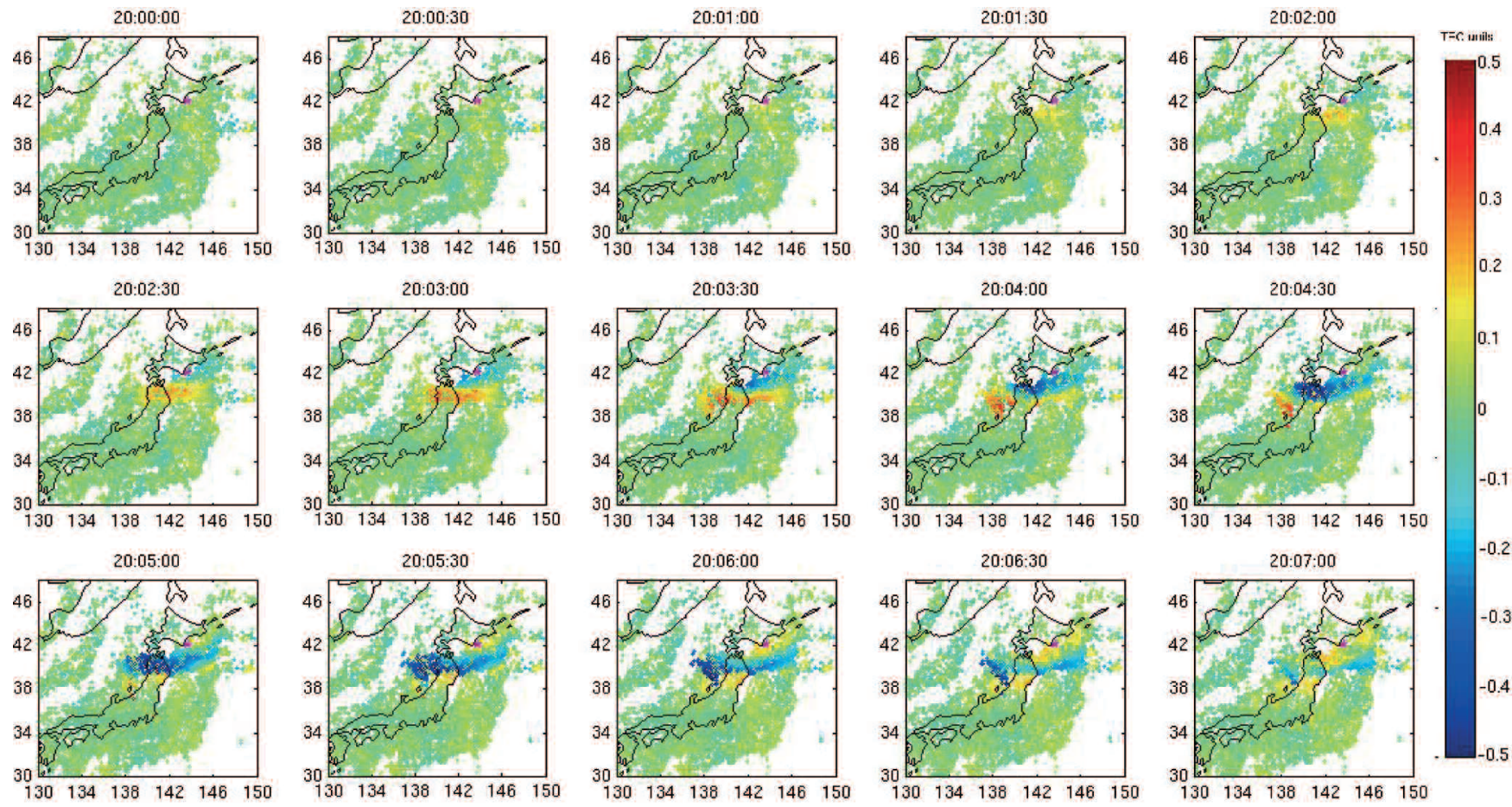

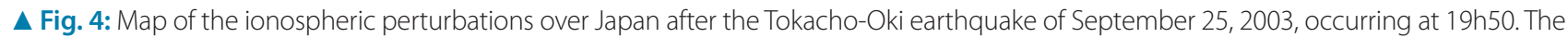
time interval between each map is $30 \mathrm{~s}$. A spectacular ionospheric perturbation is observed near the source, corresponding mainly to acoustic waves generated by the quake and appears approximately 10 minutes after. At larger distances, we also observe Rayleigh waves along the coast of the Japan Sea. The amplitudes observed are comparable to those of the Denali event and are typically 0.1 TECU peak-to-peak. 
6:20 PM

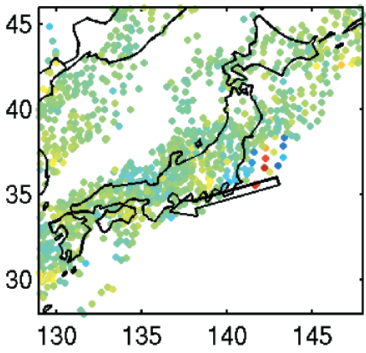

6:50 PM

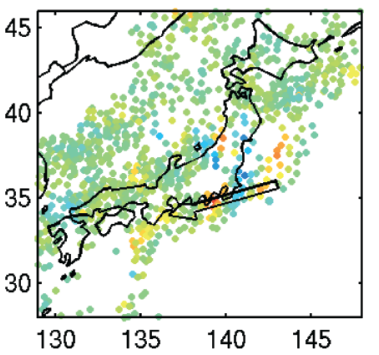

6:30 PM

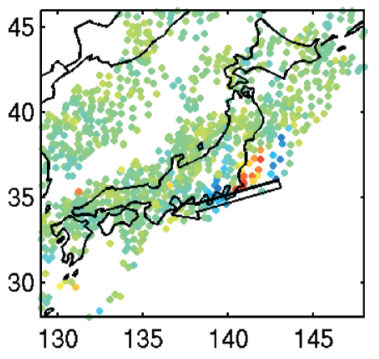

7:00 PM

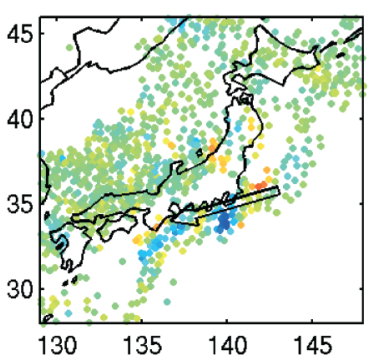

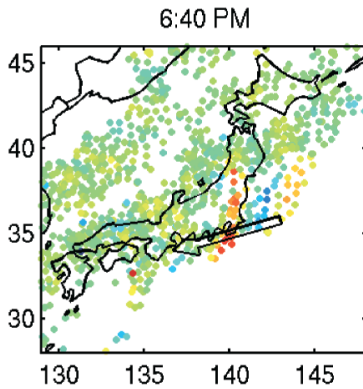

TECU

0.6

0.4

0.2

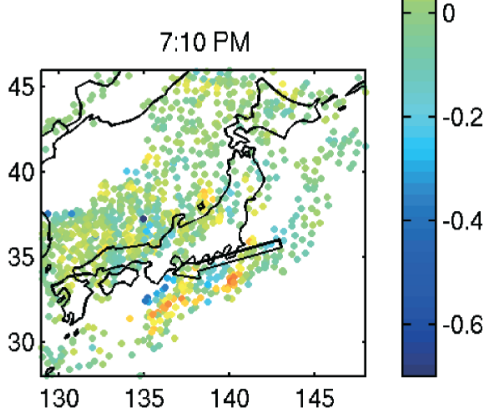

4 Fig. 5: Observed signal for the June 23, 2001 tsunami (initiated offshore Peru): TEC variations plotted at the ionospheric piercing points. A wave-like disturbance is propagating towards the coast of Honshu. This perturbation presents the expected characteristics of a tsunami-induced gravity wave, and arrives approximately at the same time as the tsunami wave itself. hours after the earthquake, with wave amplitudes between 10 and $40 \mathrm{~cm}$ (open ocean amplitude were estimated to be of $1-2 \mathrm{~cm}$ ) and dominant periods of 20 to 30 minutes. Shortly after, a large ionospheric perturbation was detected through a specific processing of data from the continuous GPS network in Japan (GEONET). Fig. 5 shows the signal observed at approximately 6:30 pm. Each dot represents the Total Electron Content (TEC) calculated from one satellite-receiver ray, corrected for ray zenithal angle and highpass filtered to remove diurnal variation. The locations of the points correspond to the intersections of the rays with the F2 peak in the ionosphere, named "piercing points". The arrival time, orientation, wavelength, velocity of the wave packet observed are consistent with what is expected for a tsunami-induced perturbation. The gigantic and dramatic Sumatra tsunami of December, 26, 2004 ( $M=9,00: 58$ UTC) confirmed the possibilities of observing tsunami ionospheric signals, and signals were detected on the Total Electronic Content (TEC) measurement on-board the TOPEX/Poseidon and JASON satellites, and on the GPS stations in Indonesia and in the India Ocean [13]. The modeling of the ionospheric signal was performed, and both the waveform and the amplitude observed by Jason and Topex has been reproduced [14]. These results confirm the interest of a real-time monitoring of the ionosphere, which could be carried out either with active microwave radar or by optical systems detecting the airglow associated with the ion recombination in the ionosphere.

In conclusion, advances in the monitoring of small-scale perturbations of the ionosphere have allowed the detection of atmospheric Rayleigh waves as well as tsunami-induced gravity waves with both ground systems based on GPS, and ionospheric sounding performed by TOPEX and JASON and Doppler sounders. These new data open exciting prospects in seismology such as the remote sensing of the Rayleigh seismic wave fronts, especially over the ocean, where the deployment of dense seismic networks is the most challenging. These prospects are also very exciting for tsunamis because they are extremely difficult to observe in the open ocean, but their associated gravity waves have a clear impact on the ionosphere and can be detected by remote sensing systems. The monitoring of the ionosphere by joint ground/space techniques, such as continuous GPS networks, over- the-horizon radar or even by a future dedicated space system, might improve our understanding of tsunami propagation in the open ocean and possibly the efficiency of the future tsunami warning systems.

\section{References}

[1] D.G. Harkrider, J. Geophys. Res., 69, 5295, 1964.

[2] P. Lognonné, C. Clévédé and H. Kanamori, Geophys. J. Int., 135, 388, 1998.

[3] H. Kanamori, and J. Mori, Geophys. Res. Lett., 19, 721, 1992.

[4] J. Artru, T. Farges, P. Lognonné, Geophys. J. Int., 158, 1067, 2004.

[5] R. Garcia, F. Crespon, V. Ducic, P. Lognonné, Geophys. J. Int, 163, 1049, 2005.

[6] More information on the Spectre project can be found at http://www.noveltis.fr/spectre. A movie showing the 3D propagation of the wave can be downloaded at: http://ganymede.ipgp.jussieu.fr/ garcia/Denali.Vcut.Az2.movie.mpg.gz

[7] V. Ducic, J. Artru and P. Lognonné, Geophys. Res. Lett., 30, 1951, 2003

[8] R. Garcia, P. Lognonné and X. Bonnin, Geophys. Res. Lett., 32, L16205, 2005,

[9] E.A. Okal, , Piatanesi, A. and P. Heinrich, J. Geophys. Res., 104, 599, 1999.

[10] Y. Tony Song, C. Ji, L.L. Fu, V. Zlotnicki, C. K. Shum, Y. Yi and V. Hjorleifsdottir, Geophys. Res. Lett, 32, 2005

[11] W.R. Peltier and C.O. Hines, J. Geophys. Res., 81, 1995, 1976.

[12] J. Artru,V. Ducic, H. Kanamori, P. Lognonné and M. Murakami , Geophy. J. Int., 160, 840, 2005.

[13] P. Lognonné , P.J. Artru, R. Garcia, F. Crespon, V. Ducic, E. Jeansou, G. Occhipinti, J. Helbert, G. Moreaux, P.E. Godet, Planet. Space. Science, in press, 2006

[14] G. Occhipinti, P. Lognonné, E. Kherani, H. Hébert, Geophys. Res. Lett, submitted, 2006. 